A 3D approach to equilibrium, stability and transport studies in RFX-mod improved regimes

This article has been downloaded from IOPscience. Please scroll down to see the full text article.

2010 Plasma Phys. Control. Fusion 52124023

(http://iopscience.iop.org/0741-3335/52/12/124023)

View the table of contents for this issue, or go to the journal homepage for more

Download details:

IP Address: 128.178.125.164

The article was downloaded on $24 / 11 / 2010$ at $13: 15$

Please note that terms and conditions apply. 


\title{
A 3D approach to equilibrium, stability and transport studies in RFX-mod improved regimes
}

\author{
D Terranova ${ }^{1}$, D Bonfiglio ${ }^{1}$, A H Boozer ${ }^{2}$, A W Cooper $^{3}$, M Gobbin ${ }^{1}$, \\ S P Hirshman ${ }^{5}$, R Lorenzini ${ }^{1}$, L Marrelli ${ }^{1}$, E Martines ${ }^{1}$, B Momo ${ }^{1}$, \\ N Pomphrey ${ }^{6}$, I Predebon ${ }^{1}$, R Sanchez $^{5}$, G Spizzo $^{1}$, M Agostini $^{1}$, \\ A Alfier $^{1}$, L Apolloni $^{1}$, F Auriemma ${ }^{1}$, M Baruzzo $^{1}$, T Bolzonella ${ }^{1}$, \\ F Bonomo ${ }^{1}$, M Brombin ${ }^{1}$, A Canton ${ }^{1}$, S Cappello ${ }^{1}$, L Carraro ${ }^{1}$, \\ R Cavazzana $^{1}$, S Dal Bello ${ }^{1}$, R Delogu ${ }^{1}$, G De Masi ${ }^{1}$, M Drevlak ${ }^{4}$, \\ A Fassina ${ }^{1}$, A Ferro ${ }^{1}$, P Franz $^{1}$, E Gaio ${ }^{1}$, E Gazza $^{1}$, L Giudicotti ${ }^{1}$, \\ L Grando ${ }^{1}$, S C Guo ${ }^{1}$, P Innocente ${ }^{1}$, D Lòpez-Bruna ${ }^{6}$, G Manduchi ${ }^{1}$, \\ G Marchiori $^{1}$, P Martin ${ }^{1}$, S Martini ${ }^{1}$, S Menmuir ${ }^{1}$, S Munaretto ${ }^{1}$, \\ L Novello ${ }^{1}$, R Paccagnella ${ }^{1}$, R Pasqualotto ${ }^{1}$, G V Pereverzev ${ }^{8}$, R Piovan $^{1}$, \\ P Piovesan ${ }^{1}$, L Piron ${ }^{1}$, M E Puiatti ${ }^{1}$, M Recchia ${ }^{1}$, F Sattin ${ }^{1}$, P Scarin ${ }^{1}$, \\ G Serianni $^{1}$, A Soppelsa ${ }^{1}$, S Spagnolo ${ }^{1}$, M Spolaore ${ }^{1}$, C Taliercio ${ }^{1}$, \\ M Valisa ${ }^{1}$, N Vianello ${ }^{1}, Z_{\text {Zang }}{ }^{1}$, A Zamengo $^{1}$, B Zaniol ${ }^{1}$, L Zanotto ${ }^{1}$, \\ P Zanca $^{1}$ and $M Z^{2}{ }^{1}$ \\ ${ }^{1}$ Consorzio RFX, Associazione EURATOM-ENEA sulla Fusione, Padova, Italy \\ 2 Department of Applied Physics and Applied Mathematics, Columbia University, New York, \\ NY, USA \\ ${ }^{3}$ EPFL, Association EURATOM-Confederation Suisse, Centre de Recherches en Physique des \\ Plasmas, Lausanne, Switzerland \\ ${ }^{4}$ Max Planck Institute für Plasmaphysik, Greifswald, Germany \\ ${ }^{5}$ ORNL Fusion Energy Division, Oak Ridge, TN, USA \\ ${ }^{6}$ Princeton Plasma Physics Laboratory, Princeton, NJ, USA \\ ${ }^{7}$ Laboratorio Nacional de Fusión, Asociación EURATOM-CIEMAT, Madrid, Spain \\ ${ }^{8}$ Max Planck Institute für Plasmaphysik, Garching, Germany \\ E-mail: david.terranova@igi.cnr.it
}

Received 21 June 2010, in final form 28 August 2010

Published 15 November 2010

Online at stacks.iop.org/PPCF/52/124023

\begin{abstract}
The full three-dimensional (3D) approach is now becoming an important issue for all magnetic confinement configurations. It is a necessary condition for the stellarator but also the tokamak and the reversed field pinch (RFP) now cannot be completely described in an axisymmetric framework. For the RFP the observation of self-sustained helical configurations with improved plasma performances require a better description in order to assess a new view on this configuration. In this new framework plasma configuration studies for RFX-mod have been considered both with tools developed for the RFP as well as considering codes originally developed for the stellarator and adapted to the RFP. These helical states are reached through a transition to a very
\end{abstract}


low/reversed shear configuration leading to internal electron transport barriers. These states are interrupted by MHD reconnection events and the large $T_{\mathrm{e}}$ gradients at the barriers indicate that both current and pressure driven modes are to be considered. Furthermore the typically flat $T_{\mathrm{e}}$ profiles in the helical core have raised the issue of the role of electrostatic and electromagnetic turbulence in these reduced chaos regions, so that a stability analysis in the correct $3 \mathrm{D}$ geometry is required to address an optimization of the plasma setup. In this view the VMEC code proved to be an effective way to obtain helical equilibria to be studied in terms of stability and transport with a suite of well tested codes. In this work, the equilibrium reconstruction technique as well as the experimental evidence of 3D effects and their first interpretation in terms of stability and transport are presented using both RFP and stellarator tools.

(Some figures in this article are in colour only in the electronic version)

\section{Introduction}

A complete three-dimensional (3D) description has always been a need for the description of stellarator plasmas. More recently the importance of 3D features has been recognized for tokamak and reversed field pinch (RFP) plasmas too.

Among others, 3D issues for tokamak concern the interaction between plasma and nonaxisymmetric magnetic field perturbation, either produced explicitly by an external action or by the plasma itself. Examples are resonant magnetic perturbations, ELM control experiments, toroidal ripple, resistive wall modes (including their active control and the issue of mode nonrigidity), neoclassical toroidal viscosity, current transport mechanisms in hybrid H-mode. 3D physics has therefore a significant impact on MHD and transport.

3D features are key to the RFP, too. Here they are often linked to the non-linear dynamics of resistive instabilities, which rule the magnetic self-organization process responsible for the configuration sustainment, and to feedback control of MHD stability. As for the latter, two European RFP devices-RFX-mod and EXTRAP T2-R — are equipped with advanced systems of feedback controlled active coils, which may produce non-axisymmetric magnetic field for simultaneous control of multiple RWMs [RWM1, RWM2] and in general of error fields.

Magnetic self-organization is an important feature of RFP dynamics [VAR08]. This nonlinear process is responsible for generating toroidal flux from the poloidal flux, which is driven by the applied toroidal loop voltage (the RFP is a purely ohmic device). As stated by the Cowling theorem [COW34], breaking of magnetic axisymmetry is a necessary condition for this process to happen. In standard, multiple helicity RFP plasmas, this is ensured by the simultaneous presence of several tearing instabilities. They drive the process, but have the rather deleterious consequence of producing broad regions of non-axisymmetric stochastic magnetic field, which leads to anomalous transport. Non-axisymmetry in this case may also appear on a gross scale when modes lock in phase, and produce toroidally localized bulging of the plasma, with severe plasma-wall interaction.

Fortunately 3D effects do not have only negative consequences in the RFP. Experiments in RFX-mod [LOR09] have indeed shown that RFP plasmas may self-organize in a single-axis helical state, dubbed SHAx, where the equilibrium is helically symmetric and is characterized by a dominant magnetic helicity (that of the innermost resonant tearing mode), with strongly 
reduced magnetic chaos and improved performance. This opens a new view on the RFP in a reactor perspective.

In RFX-mod [SON03] helical states appear regularly at high plasma current $\left(I_{\mathrm{p}} \geqslant\right.$ 1 MA) [LOR09, PZ09]: differently from the typical multiple helicity case here a single mode dominates the magnetic fluctuation spectrum with amplitude of the order of 4-5\% of the total magnetic field. The whole plasma core changes its shape, expelling the native axisymmetric magnetic axis that is replaced by a single helical axis. This helical equilibrium with iota $\iota=7$ has very low/reversed magnetic shear, leading to formation of strong internal electron transport barriers where the electron temperature $\left(T_{\mathrm{e}}\right)$ reaches $1.5 \mathrm{keV}$ (with electron density around $\left.3 \times 10^{19} \mathrm{~m}^{-3}\right)$. The large $T_{\mathrm{e}}$ gradients at the barriers $\left(6.5 \mathrm{keV} \mathrm{m}^{-1}\right)$ are similar to what is observed in the LHD stellarator [IDA08] and indicate that stability of both current and pressure driven modes should be considered. Furthermore, the typically flat $T_{\mathrm{e}}$ profile characterizing the helical core has raised the issue of the role of electrostatic and electromagnetic short-scale turbulence in these reduced chaos regions. Particle confinement is also improved [TER10] and neoclassical effects are under investigation [GOB10].

The new scenarios and perspectives opened by the RFP helical equilibria call for a 3D description of the configuration. This is needed to understand and optimize the RFP, but provides also a unique opportunity to establish and develop a knowledge basis on 3D fusion physics. The latter goal is being successfully developed in a growing partnership with the stellarator community. RFP helical states have notable commonalities with the stellarator, even though an important difference is given by the fact that RFP helical equilibria are the result of an ohmic self-organization process, when a good magnetic boundary is provided to the plasma. This means that the configuration is not completely determined by the external vacuum field, making the load assembly simpler than in a stellarator, but calling for considering also different phenomena.

With awareness of commonalities and differences, this paper reports the results of an integrated and systematic study of equilibrium and transport in helical RFP states. A significant part of it has been realized using stellarator tools adapted for the RFP, in particular the VMEC code.

The structure of the paper is as follows: in section 2 helical equilibria reconstruction will be addressed; in section 3 will be presented the comparison with experimental data and first results on stability analysis; in section 4 the role of the ohmic constraint will be considered; in section 5 particle and energy transport analysis will be addressed with the tools currently used at RFX-mod as well as some results on the role of micro-turbulence. Finally, section 6 is devoted to summary and conclusions.

\section{RFP helical equilibrium reconstruction}

Clear examples of inadequacy of axisymmetric equilibrium modelling [OS82] in describing RFX-mod (major radius $2 \mathrm{~m}$, minor radius $0.459 \mathrm{~m}$ ) experimental helical states are provided by electron temperature and density spatial profile measurements, and by the tomographic reconstruction of SXR emissivity. In helical plasmas with a magnetic fluctuation spectrum peaked on the most internally resonant mode $(m=1, n=7$, where $m$ and $n$ are, respectively, the poloidal and toroidal mode number, see figure 1 with a typical RFP $q$ profile) temperature and density profiles are non-axisymmetric along a diameter, and SXR iso-emissive surfaces indicate the presence of a helical core within an almost axisymmetric boundary.

A first equilibrium modelling approach is based on perturbative solutions of the forcebalance equation in toroidal geometry with no pressure [ZT04]. It considers the equilibrium magnetic field as a superposition of the axisymmetric background and of the eigenfunction 


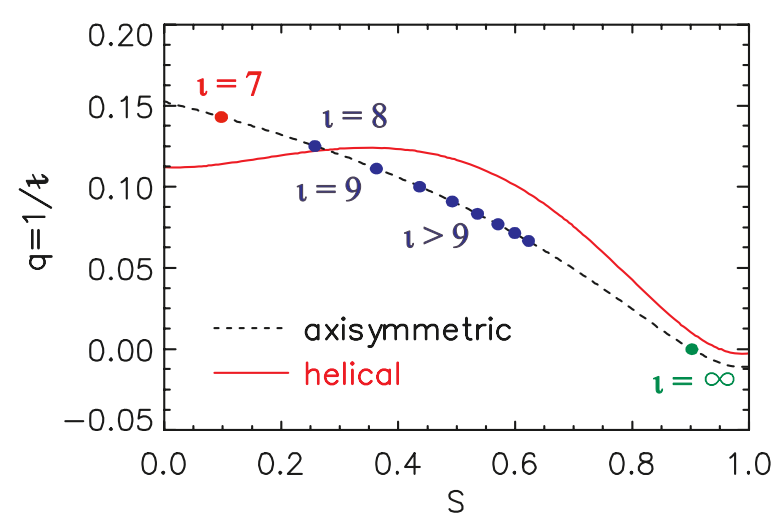

Figure 1. Safety factor profiles for an axisymmetric (dashed) and helical (continuous) RFP. Resonance radii are shown with dots. Note that the $\iota=7$ resonance is lost in the helical case. (Colour online.)

associated with the dominant mode (this single helical equilibrium-SHEq-model is described in [LOR09] and in [SHEq]). Even though the model is simple in its ingredients and neglects pressure effects, the results are good in most cases and highlight the need for a full 3D approach not only for equilibrium but also for stability and transport. Furthermore this approach allowed the determination of the safety factor profile with respect to the helical axis that is different from the monotonic profile of the axisymmetric RFP (figure 1): helical states are characterized by a null/reverse magnetic shear in the region corresponding to the temperature barrier region [ME-EPS09] and the dominant helicity is no longer resonant.

As the magnetic configuration shares similarities with the stellarator, the VMEC spectral code [HW83] was considered as a complementary equilibrium study of the helical RFP. To this end the code had to be modified in order to correctly deal with the RFP toroidal field reversal: the toroidal flux is non-monotonic and cannot be used as a flux surface label as is customary when dealing with the tokamak and stellarator configurations. The code is run providing as input data global plasma quantities such as plasma current, total toroidal flux, $q$ and pressure profiles, as well as a helical axis guess [PB09] (inferred from experimental measurements such as $T_{\mathrm{e}}$ profile or SXR tomographic reconstruction). The solution is then determined in a fixed-boundary mode, i.e. defining the shape of the last closed flux surface, LCFS. To reduce as much as possible the harmonic content, VMEC can be run with a defined toroidal machine periodicity $\left(N_{\mathrm{fp}}\right)$ so that only this mode and its harmonics are considered. The value of $N_{\mathrm{fp}}$ in a stellarator is defined by the structure of the device, while in the helical RFP it corresponds to the periodicity selected by the plasma itself as dominant mode of the spectrum and is the most internally resonant mode (in RFX-mod the $m=1, n=7$, so that $N_{\mathrm{fp}}=7$ ). While the issue of the LCFS will be considered in the next section, here we would like to underline the key role of the safety factor profile that is an input constraint to VMEC and has to be determined independently. This is done either from the SHEq equilibrium in a semi-analytical way or numerically estimated with the FLiT [INN07] or ORBIT [ORBIT] field line tracing codes following field lines as they wind around the helical axis. Both solutions provide the same result though the second one requires much more computational time.

The present RFP version of VMEC was benchmarked against a typical RFP equilibrium [ISHW09] both in the axisymmetric [ZT04] and in the helical (from SHEq) cases showing a good match between the codes when run with the same constraints and boundary conditions. Currently VMEC is being run to provide equilibria as close as possible to experimental 

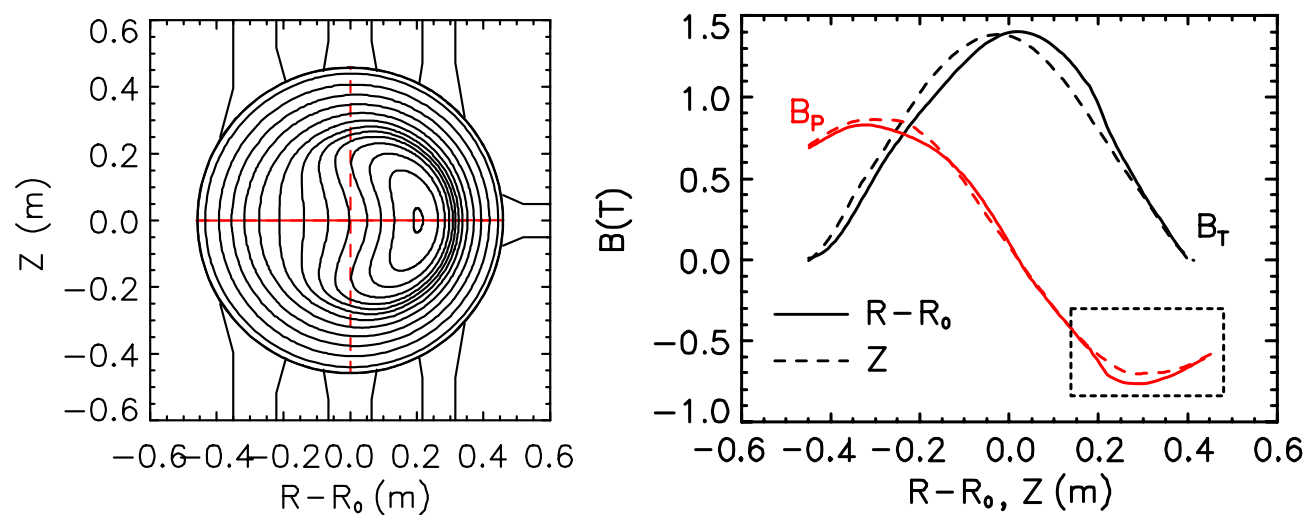

Figure 2. Left: flux surfaces structure of a helical equilibrium reconstructed with VMEC. Right: radial profiles of the toroidal and poloidal components of the magnetic field along the equatorial plane $\left(R-R_{0}\right)$ and the vertical direction $(Z)$. The region of highest asymmetries in the poloidal magnetic field is shown in the dashed rectangle. (Colour online.)

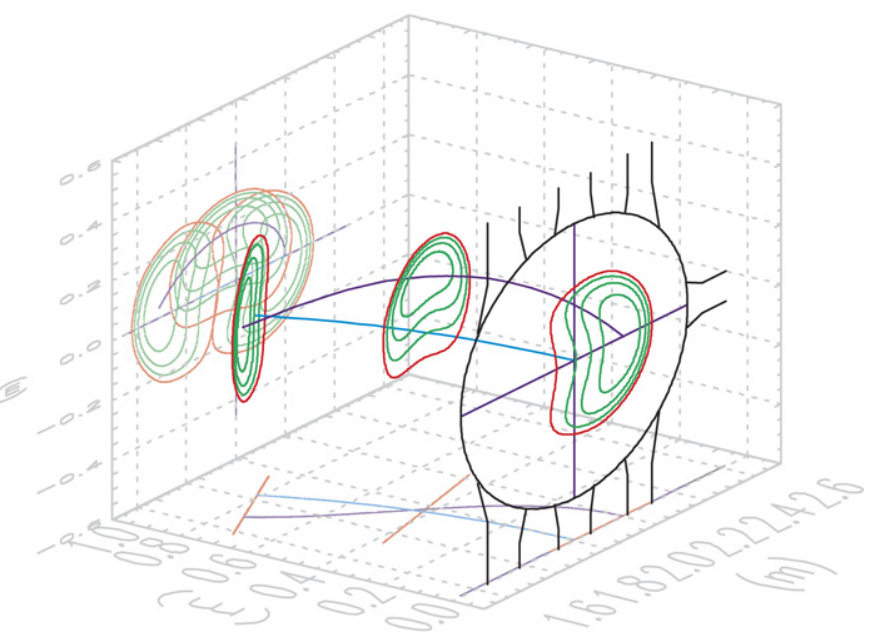

Figure 3. VMEC equilibrium and representation of the helical axis: light blue line is the vacuum vessel axis, the dark blue line starting from the centre of the bean shaped region is the helical axis. The closed contours represent the flux surfaces. The cross sections encompass half a field period. (Colour online.)

measurements, to understand the role of the boundary in helping the formation and sustainment of the helical state. As a general remark, including pressure helps the convergence of VMEC though it does not change significantly the resulting profiles. This aspect is under investigation considering different experimental situations.

A typical helical equilibrium reconstruction is shown in figure 2. The plot on the left shows the helical flux surfaces that warp the configuration in such a way that asymmetries appear in the radial profile of the toroidal and poloidal components of the magnetic field as shown in the plot on the right.

Cutaways of the 3D equilibrium are shown in figure 3 for a set of toroidal angles. Figure 4 reports a full 3D image of a helical surface in the core and the LCFS at the edge. In particular, figure 3 shows a comparison between the helical axis and the axis of the vacuum vessel: one 


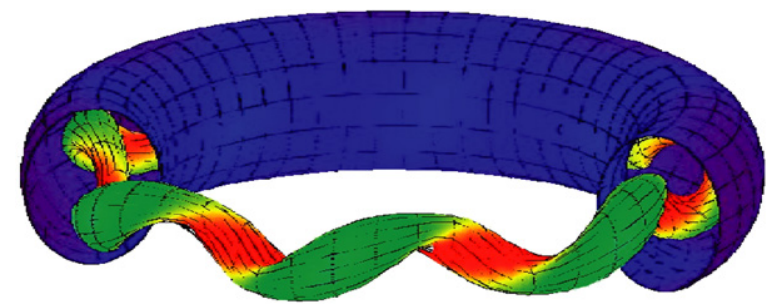

Figure 4. 3D reconstruction of the magnetic field strength: $B$ increases going from dark (blue) to light (red) colours. Two surfaces are shown: the axisymmetric LCFS and the helical core. (Colour online.)

can clearly see how the helical axis winds around the centre of the vacuum vessel as it proceeds toroidally.

\section{RFP helical equilibria and their stability}

The equilibria obtained with VMEC have to be checked against experimental measurements, as some input data are constraints while others are simply initial guesses. Apart from considering $T_{\mathrm{e}}$ and $n_{\mathrm{e}}$ profiles, another possibility is to compare the spectrum of magnetic fluctuations measured on RFX-mod with that obtained from VMEC. This information is given to VMEC through the definition of the LCFS obtained from external magnetic measurements as the sum of two components: the Shafranov shift and the local distortion of the plasma column. The latter is determined through a linear approximation from the measured spectrum of fluctuations obtained using 4 poloidal arrays of 48 toroidally distributed pick-up coils for the toroidal component and saddle coils for the radial component (note that these measurements are part of the real-time control of the magnetic boundary of RFX-mod [LUC06, MAR06]). The resulting total shift is calculated taking into account the toroidal geometry as described in [ZT04] and provided to VMEC as input. Although with this coils layout we are able to measure modes up to $n=24$, in VMEC we impose the machine periodicity to $N_{\mathrm{fp}}=7$, so that the comparison can be done only with respect to this mode and its harmonics. In particular, we retained only the main contribution to the local distortion, i.e. the one corresponding to the dominant mode.

In order to mimic the system of magnetic sensors, we used the Extender code [EXT] that allows the determination of the magnetic field on a given set of points around the plasma, starting from a VMEC equilibrium. The results for the radial component of the magnetic field are shown in figure 5 top panel, where we compare the experimental spectrum for $m=1$ and $m=0$ modes with those obtained from Extender running on the VMEC equilibrium. The comparison is quite good and could possibly be improved further adding more components to the definition of the LCFS. Note that apart from the $q$ profile, the shape of the plasma boundary is in general an important input when trying to run VMEC on an equilibrium as it can facilitate the convergence.

The significant deformation of the helical surfaces with respect to an axisymmetric case implies the existence of a magnetic field component along the radial direction. This is explicitly taken into account in the SHEq code as it corresponds to the eigenfunction of the dominant mode used in defining the helical flux [SHEq, ZT04]. In VMEC the equilibrium is the result of a global calculation so that the radial component is part of what we might call the zero-order field. As the two equilibria are determined on different flux coordinates, it is necessary to compare the reconstructed magnetic fields on a common coordinate system: in this case it is 

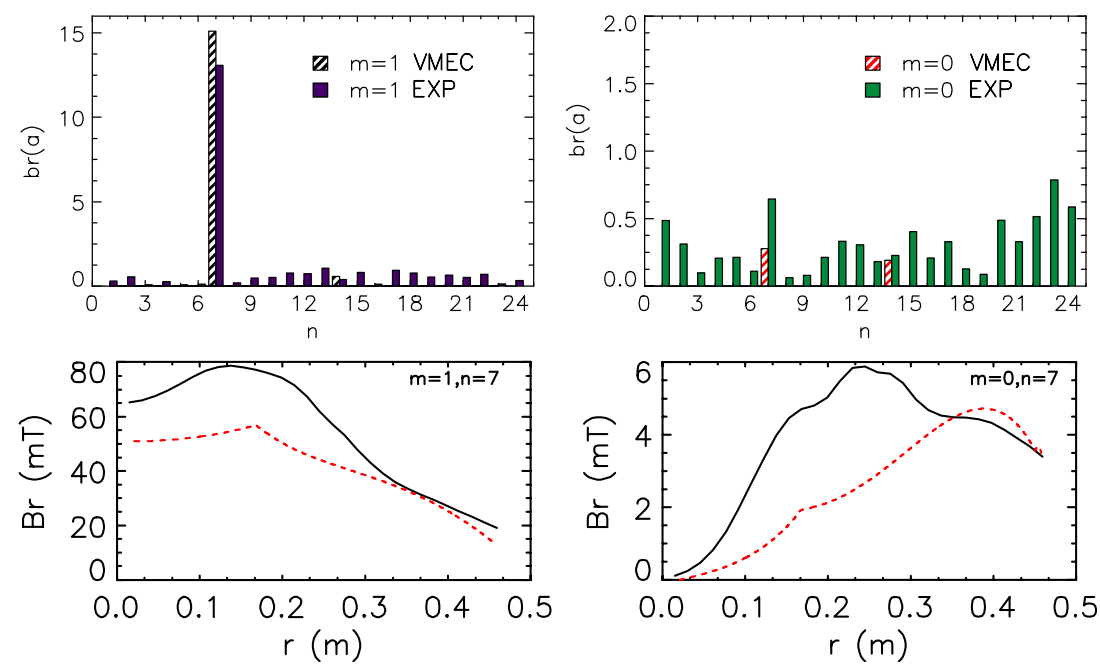

Figure 5. Top: comparison between magnetic fluctuations spectra from experimental data and from VMEC. Bottom: radial profile of the radial component of the magnetic field used in SHEq (red-dashed) and from VMEC (black-continuous). (Colour online.)

chosen as the machine system. In particular, for the VMEC case we again made use of the Extender code to obtain the quantity to be compared with SHEq. The result is shown in the bottom panel of figure 5 for the main modes characterizing the configuration, i.e. the $m=1$, $n=7$ and $m=0, n=7$. Since the equilibrium at the plasma edge is almost axisymmetric the profiles are very similar in this region. This is not the case in the helical core, where differences appear as the two codes have different approaches in solving the force-balance equation. The calculation of the eigenfunction [ZT04] assumes a thin current density layer concentrated around the resonance of the mode in the axisymmetric background field, so that a cusp appears in the profile. This is not the case for VMEC where only a diffuse current density can be modelled and the profile is therefore smooth.

The meaning of this comparison is essentially a benchmark between the codes (in both cases we are using profiles extrapolated from external measurement) and, at the same time, a starting point to understand the effect of the LCFS shape and of pressure on the radial field necessary for the helical state. A comparison with internal experimental measurements will be attempted by means of the polarimetric diagnostic in the coming experimental campaigns of RFX-mod. Indeed one should be able to measure a modulation in the poloidal component of the magnetic field as the helical core is rotating. In the right plot of figure 2 one can see that the variation in the poloidal field taken along the equatorial and vertical direction is in this case about $50 \mathrm{mT}$ (as expected comparable to the dominant eigenfunction) so that over a complete rotation a modulation twice this value should be measured.

Although the helical states observed in RFX-mod last for several energy confinement times [LOR09b], temporary back transitions through reconnection events lead to a more chaotic, lower confinement state [PZ09, ZU09]. This at the moment prevents the helical equilibrium from being fully stationary.

Numerically the stability of helical states has been addressed by means of MHD codes (3D but in cylindrical geometry) showing the importance of dissipation processes linked to both resistivity and viscosity, as well as the importance of the ratio between dominant and secondary modes [VAR08, ULq] in non-linear regimes. 
As these analyses are quite demanding already in a 3D cylindrical geometry, we decided to start addressing the issue of ideal linear stability for our helical equilibria by means of the Terpsichore code [TERPSI], looking for periodicity breaking modes, i.e. modes that have a helicity close to the one corresponding to the dominant mode. Considering kink instabilities driven by parallel current, two examples have been considered with small differences in the safety factor profile: one case with a small reversed shear and one with a null shear in the helical core. As a preliminary result, it has been found that the helical states with monotonic $q$ profile are significantly more unstable than the case with reversed shear, where the periodicity breaking modes are dominantly the $m=1, n=8$ coupled with the $m=2, n=15$ (both marginally resonant) components. As a direct extension of these results, a parametric study is currently underway to assess the role of the reversed shear region and of the resonances (also double resonances in the case of reversed magnetic shear) associated with the instability of periodicity breaking modes.

Apart from large scale instabilities and their role in the sustainment of the helical equilibrium, from a different point of view the achievement of non-chaotic regimes in a large part of the plasma volume suggests consideration also of instabilities on much smaller scales and their impact on the evolution of internal profiles and in particular on transport. This issue will be presented in section 5 .

\section{The ohmic constraint}

From a topological point of view flux surfaces in the helical RFP core bear some resemblance with a stellarator. However, as previously mentioned, an important difference is that in the RFP the 3D state is reached by an ohmic self-organization process where plasma current is playing a significant role and in particular is the only heating source. The existence of a helical state with the ohmic constraint has been modelled theoretically and numerically in the past [PUST82, CP90, CP92, FNB92] even before it was actually experimentally observed in several RFPs. For this reason one could expect that the equilibria reconstructed from experimental data should comply with the ohmic constraint. In a simple way this can be represented by the surface average of the parallel ohmic constraint relation, i.e. $\langle E \cdot B\rangle=\langle\eta(J \cdot B)\rangle$, where $\eta$ is the plasma resistivity. In a recent paper [BP09] the issue of the sustainment of an ohmic-helical state has been readdressed using the VMEC though still in a non-optimized version for the RFP. However, as the ohmic constraint is not embedded in any equilibrium model based on experimental data, it is necessary to check the constraint and how far the reconstructed solution is from an ohmic equilibrium. When assuming a constant loop voltage for the sustainment as in [FNB92], the two quantities to compare are the surface average of the contra-variant toroidal component of the magnetic field $\left\langle B^{\phi}\right\rangle$ and the parallel current density $\langle J \cdot B\rangle$. Resistivity has to be taken into account. Although in the helical core the correspondence could be reasonable, at the edge (in the region around the toroidal magnetic field reversal) the two quantities do not match and $\eta$ is smaller than in the core.

In order to see whether the discrepancy could be solved with input constraints that comply with the ohmic relation, VMEC has been run providing as input the $q$ profile from an ohmic-helical equilibrium determined with the cylindrical MHD visco-resistive SpeCyl code [SPECYL]. As a simplified case we selected a force-free equilibrium with a uniform resistivity profile and ran VMEC with a large aspect ratio since SpeCyl is a cylindrical code. In figure 6 one can see the very good agreement between the parallel current density profile predicted by VMEC and that of the ohmic equilibrium obtained from the non-linear MHD code. Although this comparison has a limited scope, it can still provide valuable information. It is indeed shown that, even without taking into account the ohmic 

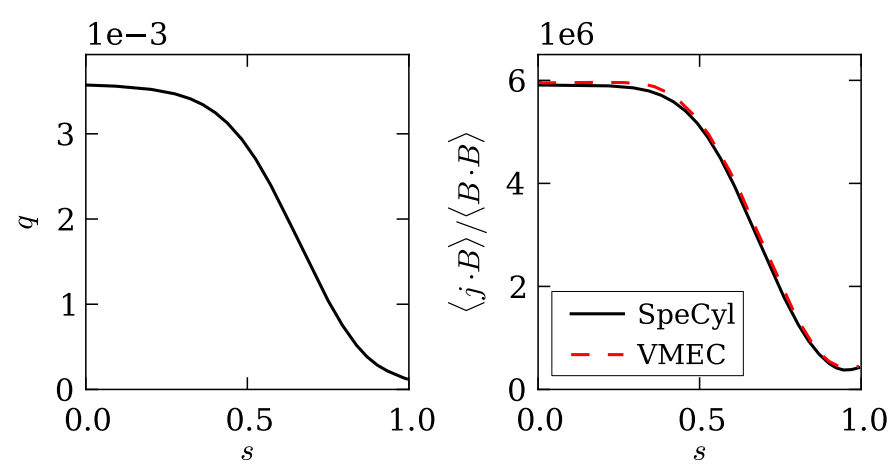

Figure 6. Left: VMEC input $q$ profile from SpeCyl. Right: SpeCyl (red-dashed) and VMEC (black-continuous) parallel current density profiles. (Colour online.)

constraint, VMEC is able to converge to an ohmic equilibrium when provided with the correct $q$ profile.

\section{Particle and energy transport}

While the effects of the helical equilibria are very evident in temperature profiles, only small changes in density profile peaking are observed [LOR06, BON09] unless a particle source is available inside the helical core (a heat source is always present through the current density channel). This happens because the penetration of neutrals from the wall is limited by the typical density and pressure profiles of RFX-mod, which feature high edge gradients. Perturbative experiments have been done in order to address the issue of particle transport, both for the main gas and impurities.

With hydrogen pellet injection experiments it was possible to determine the improvement in the global particle confinement time that is of about a factor 2 to 3 [TER10]. For those experiments a zero-order estimate of the particle diffusion coefficient $\left(D_{\mathrm{H}}\right)$ by means of a 1D simulation shows that with respect to axisymmetric states $D_{\mathrm{H}}$ decreases significantly down to values where neoclassical effects become important within the helical core. Due to the low level of collisionality, during the ablation process particles are bound to the helical shape of the plasma and density measurements are correctly interpreted only taking this into account. In figure 7 we show two Poincare plots obtained with the FLiT code [INN07] computed at the toroidal section of the pellet injector $(a)$ and of the interferometer $(b)$, and a third plot (c) with the measured time evolution of two homologous interferometric chords (blue-dashed and red-continuous lines of panel $(b)$ ) passing through the plasma core and symmetric with respect to the geometrical axis. Each field line evolves starting from the pellet trajectory so that one can reconstruct the deposition at the interferometer cross section. As the pellet enters the plasma (black dots of the Poincare plots) particles are measured mostly by the blue-dashed line of sight that is the first one to measure ablated particles. At time $t_{1}$ the pellet reaches a region where also the red-continuous line of sight detects ablated particles. At time $t_{2}$ the pellet enters the helical core (red dots of the Poincare plots) and the particles are detected only by the red-continuous line of sight until time $t_{3}$ when the two chords swap again their evolution.

This example shows that to model particle transport (and consequently a correct interpretation of density profiles) from experimental data one has to consider the correct geometry. To this end the ASTRA code [PEREV] has been considered as a tool where the metric tensor can be taken into account at least in the flux surface averaging process of the 

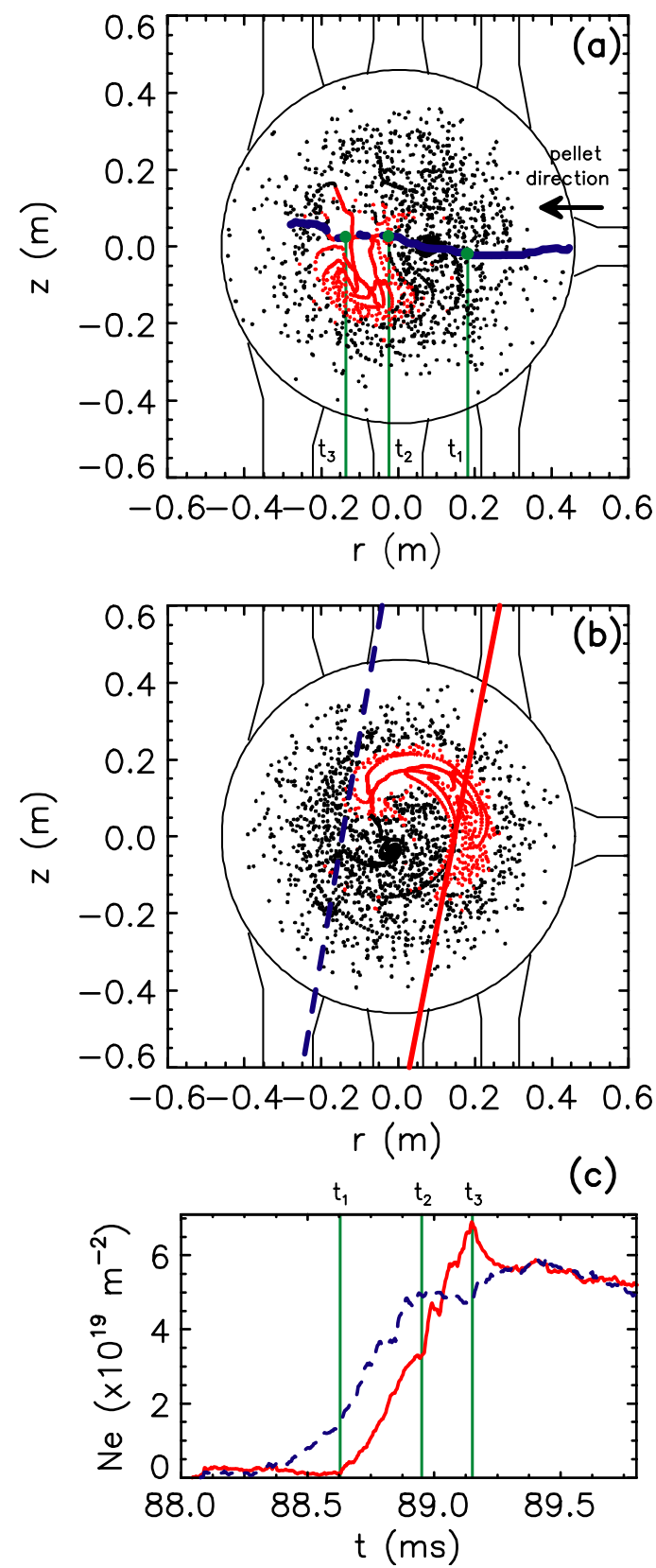

Figure 7. Poincaré plots from the FLiT code at the toroidal section of the pellet injector $(a)$ and of the interferometer $(b)$. Light (red) dots correspond to the helical core region and dark (black) dots to the surrounding plasma. (c) Time evolution of two homologous chords of the RFX-mod interferometer-dashed-blue line and red-continuous line of panel $(b)$ - passing through the plasma core. Vertical green lines correspond to 3 time instants with different pellet positions with respect to the helical core. (Colour online.) 

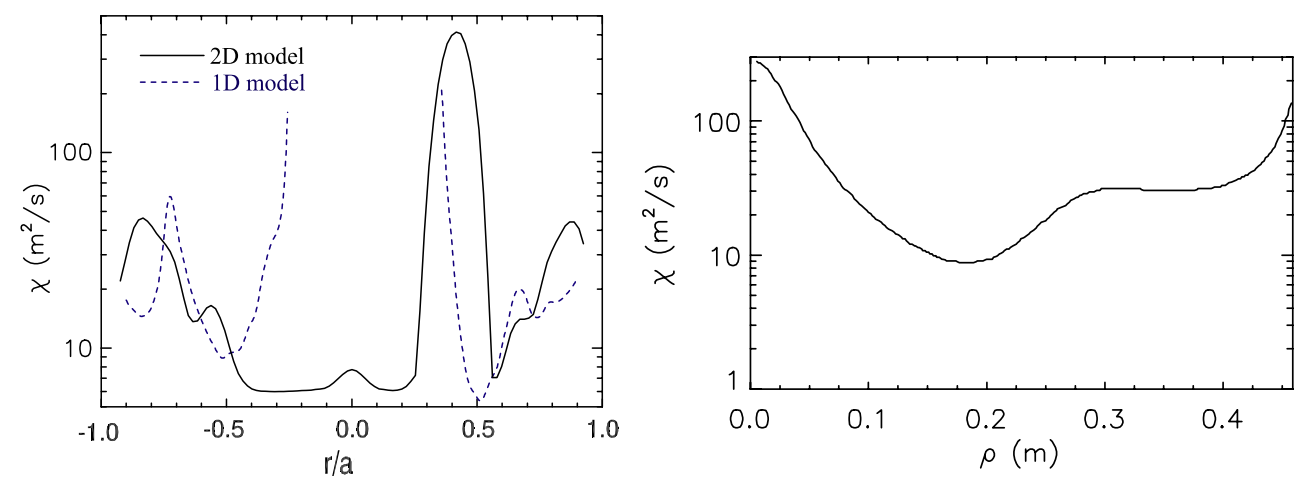

Figure 8. Calculated energy transport coefficient from experimental data with a $2 \mathrm{D}$ model (continuous) and 1D model (dashed) (left) and with ASTRA (right). (Colour online.)

main plasma quantities. Both SHEq and VMEC have been interfaced with the ASTRA code and future analysis will consider simulations of standard discharges and of pellet experiments.

Impurity transport has been addressed experimentally by means of Ni laser-blow-off (LBO) and Ne gas puffing. These experiments show that impurities (independently of their mass) behave differently from the main gas. No indication of impurity accumulation is observed [MEN10] opposite to what is generally observed in many experiments [DUX03]. However, one should consider that LBO or gas puffing particles are much less efficient in entering the plasma compared with pellet injection. All these experiments will therefore be repeated using impurity pellets in the coming campaigns. Also to understand possible similarities with the impurity hole observed in the LHD stellarator [YOS09, IDA09] due to particle expulsion.

Using a 1D model based on experimental data (i.e. temperature, density and impurity influxes), SXR radiation (including line emission) was simulated allowing for the determination of the diffusion coefficient $D$ and pinch velocity $V$. As shown in [MEN10], the penetration of impurities is always hindered by a pinch velocity directed outwards. The effect is enhanced in helical states leading to hollow profiles due to an increase in the region where a large $V$ is obtained. Also for impurities ASTRA is being considered to improve the simulation of experimental data.

In helical states temperature and density can be considered as flux functions and so resistivity and the ohmic power. This means that we can build 2D maps on a poloidal cross section defining these quantities and consequently treat in the same way also $\chi$, obtaining a 2D model [FAS09]. In figure 8 (left) we show two profiles taken on the equatorial plane assuming a standard 1D approach (dashed line) and the 2D (continuous line) model: the trend in the core is compatible with the other profiles (kinetic and magnetic) only for the second case [FAS09]. The same calculation was done with ASTRA (figure 8 right) taking into account the metrics of the system, based on a SHEq equilibrium. Both estimates agree in showing a reduction in the energy transport at the barrier with a $\chi \sim 10 \mathrm{~m}^{2} \mathrm{~s}^{-1}$. Note that neither modelling is able to correctly model the transport in the helical core (where $T_{\mathrm{e}}$ is flat) where possibly non-diffusive transport is taking place and electrostatic turbulence could be an issue [SAT10], as described later.

These results, along with the significant reduction in magnetic chaos, suggest that transport might be different from that driven by global magnetic fluctuation. Neoclassical effects and/or micro-turbulence might be ruling transport in the helical core and across the gradient of the internal barriers. In particular, the large gradients could induce the growth of instabilities 

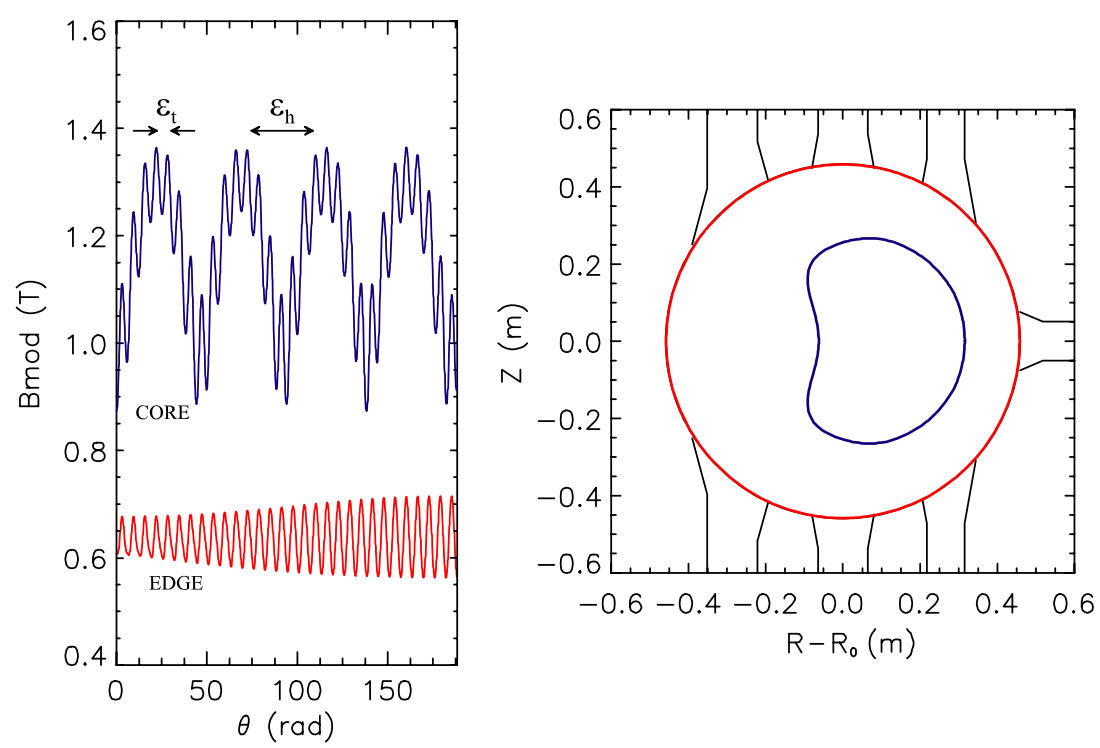

Figure 9. Left: magnetic field ripple along a field line at the edge (light-red) and in the core (darkblue) showing possible particle trapping in the helical RFP. Right: flux surfaces corresponding to the line on the left. (Colour online.)

leading to the sudden (but temporary) back transitions to the axisymmetric configuration as experimentally observed [PZ09].

The typical experimental collisionality of helical states in RFX-mod is between the banana regime and the plateau region with collisional frequency for ions around $2-3 \mathrm{kHz}$. At a basic level particle trapping can be investigated by looking at the magnetic field ripple [MYN06]. In figure 9 we show the ripple along a field line over two selected flux surfaces. The light-red surface is the LCFS representative of the axisymmetric region. The oscillations correspond to the toroidal ripple $\left(\varepsilon_{\mathrm{t}}\right.$ as in [MYN06]) and the regions of possible toroidal trapping (banana orbits). The dark-blue surface corresponds to the helical core region and the oscillations show a combination of both toroidal and helical $\left(\varepsilon_{\mathrm{h}}\right)$ ripple so that also super-banana orbits can appear. The trapped particle fraction in the helical state increases by about 10\% compared with a standard axisymmetric RFP with a negligible value of the bootstrap current [GOB09].

Neoclassical effects, and in particular super-bananas, which plague un-optimized stellarators at low collisionality, might not be a significant issue for the helical RFP. The ORBIT code shows that when trapped particles drift out of the helical core, they reach a region $(r / a \sim 0.6)$ where $\varepsilon_{\mathrm{h}}$ decreases and they become almost passing without being lost, at least for low collisionality [GOB10]. Monte Carlo transport simulations by ORBIT confirm that the ion diffusion coefficient $\left(D_{\mathrm{i}}\right)$-volume-averaged over the helical domain-versus collisionality $v$ does not show the negative $1 / v$ regime typical of stellarators. In the ideal situation where no secondary modes are considered, at the density and temperature typical of RFX-mod, ORBIT gives $D_{\mathrm{i}} \sim 0.8 \mathrm{~m}^{2} \mathrm{~s}^{-1}$ and $D_{\mathrm{e}} \sim 0.06 \mathrm{~m}^{2} \mathrm{~s}^{-1}$ for the electrons. If residual chaos is taken into account $D_{\mathrm{i}}$ slightly increases to $\sim 1 \mathrm{~m}^{2} \mathrm{~s}^{-1}$, but a greater effect can be found on the electron diffusion (due to their small mass) and $D_{\mathrm{e}}$ can become of the order of $1-2 \mathrm{~m}^{2} \mathrm{~s}^{-1}$. The different effect on the two species makes the implementation of a radial electric field necessary in order to ensure the ambipolarity constraint. This is why оRBIT has been implemented with an electrostatic potential constant on the helical flux surfaces thus generating an electric 
field $E_{r}$ perpendicular to them. This field affects mainly trapped particles that consequently can contribute to perpendicular transport [GOB10]. A complementary attempt to study the particle diffusion under the presence of an ambipolar electric field is being performed also with the DKES code [DKES1, DKES2, DKES3] adapted to the RFP, which has the advantage of focusing mainly on the local transport of the particles, without an average over the volume [GOB10].

In the final part of this section we mention the role of microinstabilities in the RFP configuration, though the effect of the 3D geometry of the system has not yet been taken into account. Among the various turbulent mechanisms acting on the ion Larmor radius scale, ion temperature gradient (ITG) turbulence has been considered as a first candidate for transport across ITBs, benchmarking the results with different approaches [GUO08, PRE10, SAT]. While in the tokamak ITG instabilities may largely affect heat transport, the RFP configuration turns out to be particularly resilient to such modes. Landau damping is the key mechanism in this respect, led by the short connection length of the RFP field. Assuming ITGs similar to the electron ones, these ITG modes are found to be subcritical in most of the experimental cases of the SHAx database. Only in some cases marginal stability is found. Compared with the tokamak, at mid-radius the threshold on the normalized logarithmic temperature gradient $\left(a / L_{T_{\mathrm{i}}}\right)$ for ITG instability is about 5 times larger. However, ITG modes are just one of a large set of possible instabilities. With the tools recently made available for the RFP, numerical analyses are devoted to a more comprehensive study on microinstabilities, e.g. for the occurrence of trapped-electron modes, and to understand the effect of a consistent impurity fraction on ITG modes. The finite plasma $\beta(\sim 2-3 \%)$ and collisionality of the RFPstabilizing factors for ITG modes-are instead at the basis of electromagnetic instabilities like the microtearing modes. Linear investigations including fluctuations in the magnetic vector potential show that such modes may contribute to the heat transport across the electron temperature barriers, consistently with the experimental estimates of the thermal conductivity. Values of $\chi_{\mathrm{e}} \sim 10 \mathrm{~m}^{2} \mathrm{~s}^{-1}$ are quasi-linearly estimated in the region corresponding to the maximum $T_{\mathrm{e}}$-gradient comparable to experimentally determined values. Due to the lower stability threshold with respect to ITG modes, $a / L_{T_{\mathrm{e}}} \sim 2$, microtearing modes become one of the candidates to trigger the electron temperature profile. These results will be readdressed in a full 3D framework in order to better understand the effect of the 3D geometry.

\section{Summary and conclusions}

A 3D description has become an important issue for the RFP configuration. Helical states are experimentally observed with characteristics that are similar to a stellarator though the system is characterized by a helical core surrounded by an axisymmetric boundary. Topologically the two configurations share some similarities, but there are also important differences: the ohmic self-organization process leading to the helical RFP implies that the plasma is playing an important role in defining the final configuration so that a comparison with experimental data is necessary to verify the goodness of the solution. The stability of the equilibrium should be investigated in the proper geometry. To this end the Terpsichore code has been used to address the issue of linear-ideal MHD perturbations and first results indicate that reversed magnetic shear in the helical core could have a stabilizing effect compared with null shear. The changes taking place in the plasma indicate that other instabilities might also be at play, on much smaller scales: their growth could be the cause of the experimentally observed back transitions from the helical state to the axisymmetric one: both the role of 3D geometry and other physical aspects (such as changes in magnetic shear and impurities) have to be addressed to assess their effect on instabilities at such small scales. 
A full description of a helical RFP equilibrium is still lacking a proper inclusion of the ohmic constraint. This is a long standing issue as several attempts were done in order to couple the force-balance equation to the ohmic constraint in $3 \mathrm{D}$ configurations. To address this problem first tests have been done by benchmarking VMEC against the visco-resistive MHD SpeCyl code in cylindrical geometry showing that indeed with the correct information in terms of input profiles the two codes converge to the same solution.

New aspects are also being considered in transport studies. Temperature gradients of helical equilibria indicate that as far as energy transport is concerned micro-turbulence could be an issue and ITG, TEM and microtearing modes are presently being studied and are possible candidates for transport on ITB.

$3 \mathrm{D}$ effects are clearly visible in density measurements, but neoclassical effects on particle transport do not seem as critical in the helical RFP as in the stellarator, due to the small drift of trapped particles (as results from the ORBIT code) and the fact that helical core is actually surrounded by an axisymmetric configuration. The DKES [DKES1, DKES2, DKES3] code is being considered for calculating local transport coefficients in order to obtain also a comparison with the global results obtained with the Monte Carlo orBIT code.

Using tools common to the stellarator and tokamak community (and VMEC guarantees integration with a broad suite of stellarator codes for transport and stability studies) the RFP will provide an additional benchmark configuration for 3D codes and will be able to reach a better integration in a shared description of all magnetic confinement configurations. Being able to test and benchmark stability and transport codes also on the helical RFP could provide further confirmation on the validity of the codes and possibly expand their applicability.

\section{Acknowledgment}

This work was supported by the European Communities under the contract of Association between EURATOM/ENEA. The views and opinions expressed herein do not necessarily reflect those of the European Commission.

(C) Euratom 2010.

\section{References}

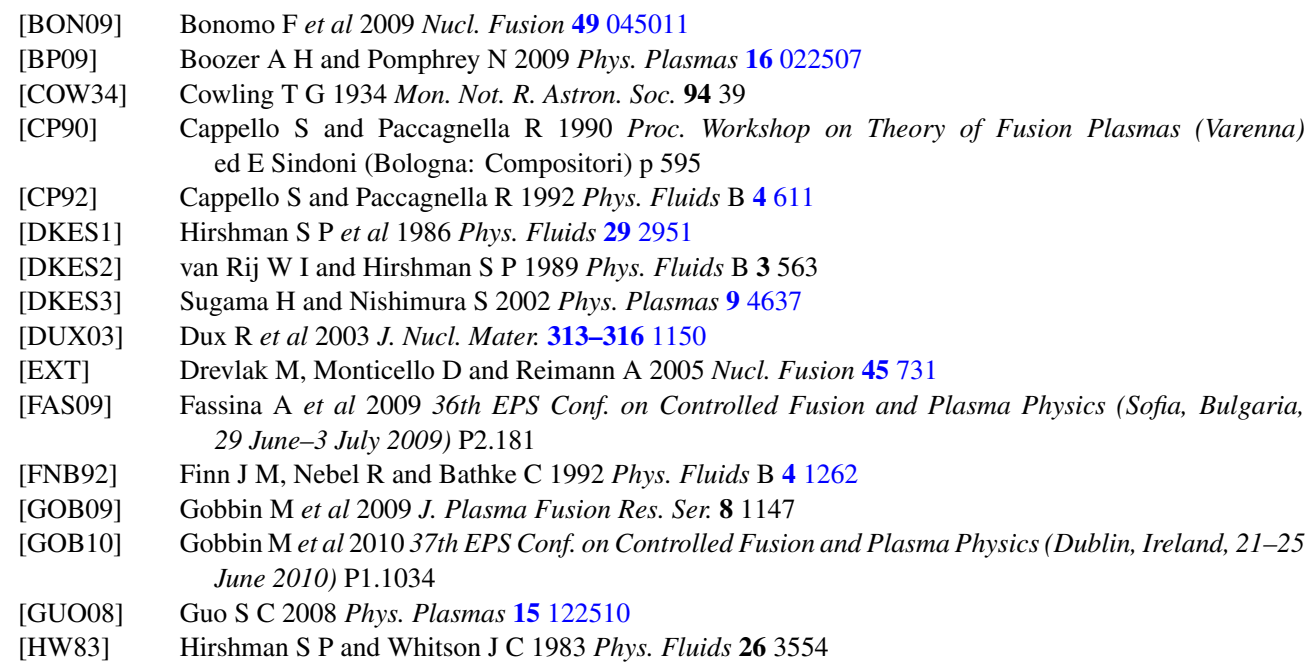


[IDA08] Ida K 2008 J. Phys.: Conf. Ser. 123012004

[IDA09] Ida K et al 2009 Phys. Plasmas 16056111

[INN07] Innocente P et al 2007 Nucl. Fusion 471092

[ISHW09] Terranova D et al 2009 17th ISHW (Princeton, NJ, 12-16 October 2009) C09

[LOR06] Lorenzini R, Auriemma F, Canton A and Carraro L 2006 Phys. Plasmas 13112510

[LOR09] Lorenzini R et al 2009 Nature Phys. 5 570-4

[LOR09b] Lorenzini R et al 2009 Phys. Plasmas 16056109

[LUC06] Lucchetta A et al 2006 21th IAEA Fus. Energy Conf. (Chengdu, China, 2006) FT/P5-1

[MAR06] Martini S et al 2006 21th IAEA Fus. Energy Conf. (Chengdu, China, 2006) EX/P7-3

[ME-EPS09] Puiatti M E et al 2009 Plasma Phys. Control. Fusion 51124031

[MEN10] Menmuir S et al 2010 Plasma Phys. Control. Fusion 52095001

[MYN06] Mynick H E 2006 Phys. Plasmas 13058102

[ORBIT] White R B and Chance M S 2004 Phys. Fluids 272455

[OS82] Ortolani S and Schnack D D 1982 Magnetohydrodynamics of Plasma Relaxation (Singapore: World Scientific)

[PB09] Pomphrey N and Boozer A 2009 ReNew Workshop: Theme V March 2009 (http://www.pppl.gov/ conferences/ReNeW/T5Workshop/Wed_am_pres/Helical_pomphrey.pdf)

[PEREV] Pereversev G et al 2002 Max Planck Institut für Plasmaphysik Report IPP 5/98, Garching, February 2002

[PRE10] Predebon I, Angioni C and Guo S C 2010 Phys. Plasmas 17012304

[PUST82] Pustovitov V D 1982 Pis'ma Zh. Eksp. Teor. Fiz. 353

Pustovitov V D 1982 JETP Lett. 351

[PZ09] Piovesan P et al 2009 Nucl. Fusion 49085036

[RWM1] Brunsell P R et al 2004 Phys. Rev. Lett. 93225001

[RWM2] Paccagnella R et al 2006 Phys. Rev: Lett. 97075001

[SAT] Sattin F, Garbet X and Guo S C 2010 Plasma Phys. Control. Fusion 52105002

[SAT10] Sattin F, Vianello N, Lorenzini R, Gobbin M and Bonomo F Plasma Phys. Control. Fusion submitted

[SHEq] Momo B et al 201037 th EPS Conf. on Controlled Fusion and Plasma Physics (Dublin, Ireland, 21-25 June 2010) P4.147

[SON03] Sonato P et al 2003 Fusion Eng. Des. 66161

[SPECYL] Cappello S and Biskamp D 1996 Nucl. Fusion 36571

[TER10] Terranova D et al 2010 Nucl. Fusion 50035006

[TERPSI] Cooper W A et al 2006 Fusion Sci. Technol. 50245

[ULq] Bonfiglio D et al 2008 Nucl. Fusion 48115010

[VAR08] Cappello S 2008 Theory of Fusion Plasmas, AIP Conf. Proc. 271069

[YOS09] Yoshinuma M et al 2009 Nucl. Fusion 49062002

[ZT04] Zanca P and Terranova D 2004 Plasma Phys. Control. Fusion 461115

[ZU09] Zuin M et al 2009 Plasma Phys. Control. Fusion 51035012 